\title{
Evaluation of three enzyme immunoassays and a nucleic acid amplification test for the diagnosis of Clostridium difficile-associated diarrhea at a university hospital in Brazil
}

\author{
Rodrigo Otávio Silveira Silva ${ }^{[1]}$, Eduardo Garcia Vilela ${ }^{[2]}$, Monique Silva Neves ${ }^{[1]}$ \\ and Francisco Carlos Faria Lobato ${ }^{[1]}$
}

[1]. Faculdade de Medicina Veterinária, Universidade Federal de Minas Gerais, Belo Horizonte, MG. [2]. Faculdade de Medicina, Universidade Federal de Minas Gerais, Belo Horizonte, MG.

\begin{abstract}
Introduction: Despite the known importance of Clostridium difficile as a nosocomial pathogen, few studies regarding Clostridium difficile infection (CDI) in Brazil have been conducted. To date, the diagnostic tests that are available on the Brazilian market for the diagnosis of CDI have not been evaluated. The aim of this study was to compare the performances of four commercial methods for the diagnosis of CDI in patients from a university hospital in Brazil. Methods: Three enzyme immunoassays (EIAs) and one nucleic acid amplification test (NAAT) were evaluated against a cytotoxicity assay (CTA) and toxigenic culture (TC). Stool samples from 92 patients with suspected CDI were used in this study. Results: Twenty-five (27.2\%) of 92 samples were positive according to the CTA, and $23(25 \%)$ were positive according to the TC. All EIAs and the NAAT test demonstrated sensitivities between 59 and $68 \%$ and specificities greater than $91 \%$. Conclusions: All four methods exhibited low sensitivities for the diagnosis of CDI, which could lead to a large number of false-negative results, an increased risk of cross-infection to other patients, and overtreatment with empirical antibiotics.
\end{abstract}

Keywords: Nosocomial. Pseudomembranous colitis. Diagnosis. ELISA.

\section{INTRODUCTION}

Clostridium difficile was first isolated in 1935, but this microorganism was only recognized as a human pathogen in the late 1970s. Currently, Clostridium difficile infection (CDI) is recognized as the main cause of nosocomial diarrhea. In the last few years, the emergence of highly virulent strains of C. difficile in several countries ${ }^{1}$ and cases in outpatients with no history of antibiotic therapy have been reported, demonstrating the need for further studies related to the diagnosis and control of this pathogen ${ }^{2}$.

For many years, the detection of A/B toxins in feces via a cell cytotoxicity assay (CTA) was considered the gold standard method for the diagnosis of $\mathrm{CDI}^{2}$. More recently, studies have shown that toxigenic culture (TC) is more sensitive; therefore, it has been used as the new gold standard method ${ }^{3}$. However, both techniques are time consuming, laborious, and require

Address to: Dr. Rodrigo Otávio Silveira Silva. EV/UFMG. Av. Antônio Carlos 6627, 31270-901 Belo Horizonte, MG, Brasil.

Phone/Fax: 5531 3409-2103

e-mail: rodrigo.otaviosilva@gmail.com

Received 8 May 2014

Accepted 31 July 2014 trained personnel. Thus, commercial enzyme immunoassays (EIA) are currently the most widely used techniques for the diagnosis of $\mathrm{CDI}^{3}$.

Other potential options that have been widely cited include commercial real time polymerase chain reaction (PCR) kits and nucleic acid amplification tests (NAATs). Studies investigating these assays found that they exhibit high sensitivity, suggesting that they could be useful for screening patients with $\mathrm{CDI}^{2,4}$. Currently, the main limitation of these kits is their high cost, which is typically up to ten times more expensive than EIAs ${ }^{5}$.

Despite the known importance of $C$. difficile as a nosocomial pathogen, few studies have been conducted regarding CDI in Brazil. To date, the diagnostic tests that are available on the Brazilian market for the diagnosis of CDI have not been evaluated. Therefore, the aim of this study was to compare the performance of three commercial EIAs and one NAAT for the diagnosis of diarrhea attributed to $C$. difficile versus cytotoxicity and toxigenic culture assays.

\section{METHODS}

Between December 2011 and June 2013, 92 stool samples were collected from patients at the University Hospital of the Federal University of Minas Gerais. All samples were obtained from inpatients with suspected $C$. difficile-associated diarrhea. Specimens were collected in sterile containers, and aliquots 
were held at $-20^{\circ} \mathrm{C}$ until all tests were performed. All procedures were previously approved by the Research Ethics Committee of the School of Medicine of Federal University of Minas Gerais (CAAE - 0710.0.203.0000.11).

Cytotoxicity assays were performed using Vero (African green monkey kidney) cells (ATCC CCL 81) ${ }^{6}$. Briefly, fecal samples were diluted 1:4 in phosphate-buffered saline $(\mathrm{pH}$ 7.0 ) and centrifuged at $3,000 \mathrm{xg}$ for $5 \mathrm{~min}$ at $4^{\circ} \mathrm{C}$. The resulting supernatants were filtered through a $0.22-\mu \mathrm{m}$ pore size filter and subject to 2 fold dilutions until a dilution of 1:1024 was achieved. Serial dilutions and parallel samples with Clostridium sordellii antitoxin were added onto Vero cell monolayers. The cells were examined after $24 \mathrm{~h}$ of incubation at $37^{\circ} \mathrm{C}$ in a $5 \% \mathrm{CO}_{2}$ incubator. A specimen was considered positive by the CTA if at least $90 \%$ of cells were rounded and if the effects were neutralized by an antitoxin at the same dilution in a parallel sample.

For toxigenic culture, equal volumes of stool samples and $96 \%$ ethanol (v/v) were mixed. After incubation for 30min at room temperature, $50 \mu 1$ aliquots were inoculated on plates containing cycloserine-cefoxitin fructose agar supplemented with $7 \%$ horse blood and $0.1 \%$ sodium taurocholate ${ }^{6}$. After anaerobic incubation at $37^{\circ} \mathrm{C}$ for $96 \mathrm{~h}$, all colonies with suggestive morphologies were subjected to a previously described multiplex-PCR protocol involving a housekeeping gene $(t p i)$, toxins $\mathrm{A}(t c d A)$ and $\mathrm{B}(t c d B)$, and a binary toxin gene $(c d t B)^{7}$. All strains positive for $t c d A$ or/and $t c d B$ were considered toxigenic. In addition, all toxigenic isolates from the PCR were tested by CTA for in vitro toxin production as previously described ${ }^{8}$.

The following three commercial EIAs for A/B toxin detection were tested: $C$. difficile Tox A/B II (Techlab Inc., USA), Remel ProSpecT $C$. difficile Toxin A/B (Oxoid, UK), and Ridascreen Clostridium difficile toxins A/B (R-Biopharm, Germany). In addition, one commercial NAAT kit (Simplexa ${ }^{\mathrm{TM}}$ C. difficile Universal Direct Kit, Focus Diagnostics, USA) that directly detects the $t c d B$ gene in stool samples was also tested. All EIAs and the NAAT were performed according to the manufacturers' recommendations. The sensitivity, specificity, positive predictive value (PPV), negative predictive value (NPV), and $95 \%$ confidence interval were calculated for each EIA and NAAT versus CTA and TC. Additionally, the kappa coefficient ${ }^{9}$ was calculated to compare the CTA and TC (Stata 12, College Station, Texas, USA).

\section{RESULTS}

From the 92 sampled patients, 25 (27.2\%) were positive according to CTA. C. difficile was isolated from 29 (31.5\%) samples, of which six isolates were considered non-toxigenic and $23(25 \%)$ were toxigenic according to PCR. The kappa concordance between TC and CTA was 0.71 (95\% confidence interval, 0.51-0.9). The three tested EIAs and the NAAT exhibited sensitivities between 59 and $68 \%$ and specificities greater than 91\% (Table 1).
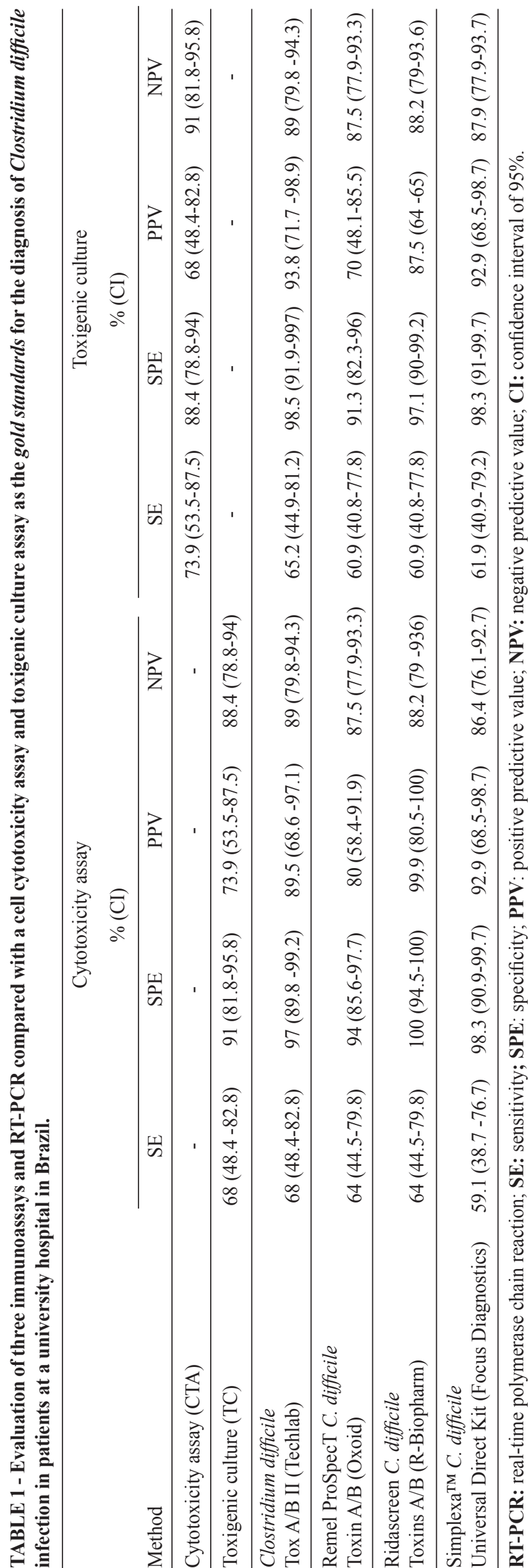


\section{DISCUSSION}

Approximately ten years ago, it was believed that the CTA demonstrated sensitivity and specificity values greater than $99 \%$ and was thus considered the primary technique to diagnose $\mathrm{CDI}^{10,11}$. However, when compared with toxigenic culture, the sensitivities of CTA protocols range between 60 and $86 \%$ with specificities greater than $90 \%{ }^{5}$. Corroborating these findings, the CTA protocol used in the present study demonstrated a sensitivity of $73.9 \%$ (Table 1). It is also important to note that no standard protocol exists for CTAs. In this study, Vero cells were used; this cell line is considered to be the most sensitive to A/B toxins, and a similar CTA protocol was used successfully in previous studies ${ }^{6,8,12-15}$.

In the TC assay, 23 (25\%) patients were positive for toxigenic strains. Of these patients, $15(65.2 \%)$ were $\mathrm{A}^{+} \mathrm{B}^{+} \mathrm{CDT}^{-}$, six $(26.1 \%)$ were $\mathrm{A}^{+} \mathrm{B}^{+} \mathrm{CDT}^{+}$, and two $(8.7 \%)$ were $\mathrm{A}^{-} \mathrm{B}^{+} \mathrm{CDT}$. All of the $C$ difficile isolates considered to be toxigenic by PCR produced toxins A and B in vitro. Conversely, the TC protocol had a sensitivity that was lower $(73.9 \%)$ than the values commonly reported in previous studies, which have recorded sensitivities close to $100 \% \%^{2,16}$. No standard method for TC assays with $C$. difficile is available, making it difficult to compare results from other studies. A wide variety of media and differences in isolation protocols, such as the use of shock alcohol and variations in incubation time, are common ${ }^{5,10}$. In this study, we opted for a simple isolation protocol, which would be more applicable for diagnosis when compared with previous methods ${ }^{17}$. It is well known that some strains of $C$. difficile may not grow due to susceptibility to either one or both antibiotics used in the medium ${ }^{18}$. Recently, Malik et al. ${ }^{19}$ demonstrated that antibiotics used in selective media are responsible for the addition of stress in the recovery of $C$. difficile spores, which might reduce isolation rates. In addition, the use of CCFA, even with supplemental taurocholate, can result in a variable sensitivity for the recovery of $C$. difficile spores compared with protocols that use pre-enrichment broth before plating on selective agar ${ }^{19}$. All of these factors potentially contributed to the sensitivity exhibited in the TC assay in this study. It should be noted that, even with a TC protocol with sensitivity slightly lower than commonly reported, the concordance (kappa) between TC and CTA was 0.71 . This value could be considered reflective of substantial agreement ${ }^{9}$ and is similar to that reported by Keessen et al. ${ }^{13}$

It also important to note that asymptomatic colonization by toxigenic or non-toxigenic $C$. difficile is considered rare in healthy adults. However, asymptomatic colonization is estimated to occur in approximately 9 to $20 \%$ in some groups, such as residents of long-term care facilities ${ }^{2,5,20}$. The detection of the $t c d A$ and $t c d B$ genes by PCR may decrease the occurrence of false-positive results by TC after patients with non-toxigenic strains have been identified as not positive; in the present study, four (4.3\%) patients who were suspected of having nosocomial diarrhea caused by $C$. difficile were negative for $\mathrm{A} / \mathrm{B}$ toxins and carried non-toxigenic strains. Conversely, eight (8.7\%) patients were positive for $\mathrm{TC}$ but negative for $\mathrm{A} / \mathrm{B}$ toxins by $\mathrm{CTA}$ and the three EIAs tested. In this case, a false-positive result should be considered a possibility. According to Peterson et al. ${ }^{20}$, the false-positive rate for TC is approximately $10 \%$. Therefore, comparisons of tests for the diagnosis CDI should be performed in parallel with CTA and TC as the gold standard methods.

Due to its ease of use, low cost and the fast turnaround for results, EIA remains the most commonly used test for the diagnosis of CDI in humans and animals worldwide ${ }^{10,12,20}$. However, the three EIAs tested in this study demonstrated low sensitivities ranging from 61 to $68 \%$. This result is similar to previous studies with EIA kits and rapid tests, which reported sensitivities between 50 and $77 \%$ and specificities of approximately $90 \% 0^{5,21,22}$.

The NAAT tested in this study also exhibited a low sensitivity (less than 62\%). This result contrasts with previous studies that have used other NAAT tests, which reported specificities and sensitivities greater than $90 \% \%^{5,21-23}$. Compared with other kits on the market, the main advantage of the NAAT tested in this study is the lack of a protocol for deoxyribonucleic acid (DNA) extraction from stool samples, which could save time and labor. Briefly, in this test, the contents of a clinical specimen swab are immersed in a lysis solution, followed by heating and centrifugation. The resulting supernatant is directly used as a DNA template in a PCR reaction. Some authors have stated that false-negative results in the NAAT are primarily caused by the presence of gene copy numbers below the detection limits of the test $\mathrm{t}^{20-24}$. Considering the number of false negative results obtained in the present work, the potential use of a more accurate extraction protocol should be considered for better performance of diagnostic tests; this modification might increase the number of gene copies and also reduce the presence of inhibitors in the sample reactions.

One sample exhibited an undetermined result in the NAAT. An additional unfrozen aliquot of this sample was retested, but an undetermined result was obtained. Thus, this sample was not considered when calculating the sensitivity, specificity, and positive and negative predictive values for the NAAT test. This stool sample was also positive in TC $\left(\mathrm{A}^{-} \mathrm{B}^{+} \mathrm{CDT}^{-}\right)$ but negative for $\mathrm{A} / \mathrm{B}$ toxins in the CTA and in all three EIAs tested. Undetermined results are mainly caused by the presence of inhibitors in the stool sample 5 . It appears that this is not a common event, given that it occurred in only one sample out of $81(1.2 \%)$ stool samples tested in the NAAT. This rate is similar to previous reports with other NAATs ${ }^{5,21}$.

Several alternatives have been proposed to improve the diagnosis of CDI. In contrast to our results, the vast majority of commercial NAATs demonstrated high sensitivities in previous studies, but their high costs prevent their wide use $e^{2,5,21}$. At institutions where EIAs are still used for the diagnosis of CDI, some authors have suggested the submission of more than one sample from the same patient, but this practice remains controversial given that some studies have demonstrated that multiple samples do not significantly increase the positive predictive value of the test and may even increase the rate of false positives ${ }^{5,25,26}$. Some authors have also suggested that an 
algorithm with at least two steps is required for the accurate diagnosis of CDI in humans, but there is still no consensus on which tests should be used in each step s, $16,22,27$

In conclusion, all four methods tested in this study demonstrated low sensitivities for the diagnosis of CDI under the conditions tested. This might lead to a large number of false-negative results, which could increase the risk of crossinfection to other patients and also overtreatment with empirical antibiotics ${ }^{21}$. The present study reinforces the need for research focusing on new methods or algorithms for the diagnosis of C. difficile, given that control of CDI is nearly impossible and the risk of its dissemination is high without a secure diagnosis.

\section{CONFLICT OF INTEREST}

The authors declare that there is no conflict of interest.

\section{FINANCIAL SUPPORT}

Conselho Nacional de Desenvolvimento Cientifico e Tecnológico (CNPq), Fundação de Amparo a Pesquisa do Estado de Minas Gerais (FAPEMIG) and Pró-Reitoria de PesquisaUniversidade Federal de Minas Gerais (PRPq-UFMG).

\section{REFERENCES}

1. Balassiano IT, Yates EA, Domingues RM, Ferreira EO. Clostridium difficile: a problem of concern in developed countries and still a mystery in Latin America. J Med Microbiol 2012; 61:169-179.

2. Silva Júnior M. Recent changes in Clostridium difficile infection. Einstein 2012; 10:105-109.

3. René P, Frenette CP, Schiller I, Dendukuri N, Brassard P, Fenn S, et al. Comparison of eight commercial enzyme immunoassays for the detection of Clostridium difficile from stool samples and effect of strain type. Diagn Microbiol Infect Dis 2012; 73:94-96.

4. Viala C, Le Monnier A, Maataoui N, Rousseau C, Collignon A, Poilane I. Comparison of commercial molecular assays for toxigenic Clostridium difficile detection in stools: BD GeneOhm Cdiff, XPert C. difficile and illumigene $C$. difficile. J Microbiol Methods 2012; 90:83-85.

5. Humphries RM. Laboratory Tests for the Diagnosis of Clostridium difficile Infections. Clinic Microbiol Newsletter 2012; 34:151-157.

6. Silva RO, Ribeiro MG, Palhares MS, Borges AS, Maranhão RP, Silva MX, et al. Detection of A/B toxin and isolation of Clostridium difficile and Clostridium perfringens from foals. Equine Vet J 2013; 45:671-675.

7. Silva ROS, Salvarani FM, Cruz Junior ECC, Pires PS, Santos RLR, Assis RA, et al. Detection of toxins A/B and isolation of Clostridium difficile from piglets in Brazil. Ciênc Rural 2011; 41:1130-1135.

8. Medina-Torres CE, Weese JS, Staempfli HR. Validation of a commercial enzyme immunoassay for detection of Clostridium difficile toxins in feces of horses with acute diarrhea. J Vet Intern Med 2010; 24:628-632.

9. Landis JR, Koch GG. The measurement of observer agreement for categorical data. Biometrics 1977; 33:159-174

10. Delmée M. Laboratory diagnosis of Clostridium difficile disease. Clin Microbiol Infect 2001; 7:411-416.
11. Wilcox MH. Laboratory diagnosis of Clostridium difficile infection: in a state of transition or confusion or both? J Hosp Infect 2011; 79:1-3.

12. van den Berg RJ, Bruijnesteijn van Coppenraet LS, Gerritsen HJ, Endtz HP, van der Vorm ER, Kuijper EJ. Prospective multicenter evaluation of a new immunoassay and real-time PCR for diagnosis of Clostridium difficile-associated diarrhea in hospitalized patients. J Clin Microbiol 2005; 43:5338-5340.

13. Keessen EC, Hopman NE, van Leengoed LA, van Asten AJ, Hermanus C, Kuijper EJ, et al. Evaluation of four different diagnostic tests to detect Clostridium difficile in piglets. J Clin Microbiol 2011; 49:1816-1821.

14. Silva ROS, Moreira FM, Rezende JV, Pires PS, Maranhão RPA, Palhares MS, et al. First confirmed case of Clostridium difficileassociated diarrhea in foals in Brazil. Ciência Rural 2012; 42:498-500.

15. Silva RO, D'elia ML, Magalhães Soares DF, Cavalcanti ÁR, Leal RC, Cavalcanti G, et al. Clostridium difficile-associated diarrhea in an ocelot (Leopardus pardalis). Anaerobe 2013; 20:82-84.

16. Shin BM, Kuak EY, Lee EJ, Songer JG. Algorithm combining toxin immunoassay and stool culture for diagnosis of Clostridium difficile infection. J Clin Microbiol 2009; 47:2952-2956.

17. Sharp SE, Ruden LO, Pohl JC, Hatcher PA, Jayne LM, Ivie WM. Evaluation of the C.Diff Quik Chek Complete Assay, a new glutamate dehydrogenase and $\mathrm{A} / \mathrm{B}$ toxin combination lateral flow assay for use in rapid, simple diagnosis of Clostridium difficile disease. J Clin Microbiol 2010; 48:2082-2086.

18. Songer JG, Uzal FA. Clostridial enteric infections in pigs. J Vet Diagn Invest 2005; 17:528-536.

19. Malik DJ, Patel KV, Clokie MR, Shama G. On the difficulties of isolating Clostridium difficile from hospital environments. J Hosp Infect 2013; 84:181-183.

20. Peterson LR, Manson RU, Paule SM, Hacek DM, Robicsek A, Thomson Jr RB, et al. Detection of toxigenic Clostridium difficile in stool samples by real-time polymerase chain reaction for the diagnosis of $C$. difficileassociated diarrhea. Clin Infect Dis 2011a; 45:1152-1160.

21. Eastwood K, Else P, Charlett A, Wilcox M. Comparison of nine commercially available Clostridium difficile toxin detection assays, a real-time PCR assay for $C$. difficile $t c d B$, and a glutamate dehydrogenase detection assay to cytotoxin testing and cytotoxigenic culture methods. J Clin Microbiol 2009; 47:3211-3217.

22. Alcalá L. Laboratory tests for diagnosis of Clostridium difficile infection: past, present, and future. Enferm Infecc Microbiol Clin 2013; 31:65-67.

23. Stamper PD, Alcabasa R, Aird D, Babiker W, Wehrlin J, Ikpeama I, et al. Comparison of a commercial real-time PCR assay for $t c d B$ detection to a cell culture cytotoxicity assay and toxigenic culture for direct detection of toxin-producing Clostridium difficile in clinical samples. J Clin Microbiol 2009; 47:373-378.

24. Novak-Weekley SM, Marlowe EM, Miller JM, Cumpio J, Nomura JH, Vance $\mathrm{PH}$, et al. Clostridium difficile testing in the clinical laboratory by use of multiple testing algorithms. J Clin Microbiol 2010; 48:889-893.

25. Litvin M, Reske KA, Mayfield J, McMullen KM, Georgantopoulos P, Copper S, et al. Identification of a pseudo-outbreak of Clostridium difficile infection (CDI) and the effect of repeated testing, sensitivity, and specificity on perceived prevalence of CDI. Infect Control Hosp Epidemiol 2009; 30:1166-1171.

26. Gade R, Turett G. The utility of repeated stool toxin testing for diagnosing Clostridium difficile colitis. South Med J 2009; 102: 1007-1009.

27. Peterson LR, Mehta MS, Patel PA, Hacek DM, Harazin M, Nagwekar PP, et al. Laboratory testing for Clostridium difficile infection: light at the end of the tunnel. Am J Clin Pathol 2011; 136:372-380. 